\title{
When objects lose their meaning: What happens to their use?
}

\author{
SASHA BOZEAT \\ MRC Cognition and Brain Sciences Unit, Cambridge, England \\ MATTHEW A. LAMBON RALPH \\ University of Manchester, Manchester, England \\ KARALYN PATTERSON \\ MRC Cognition and Brain Sciences Unit, Cambridge, England \\ and \\ JOHN R. HODGES \\ MRC Cognition and Brain Sciences Unit, Cambridge, England \\ and Addenbrooke's Hospital, Cambridge, England
}

\begin{abstract}
The 8 patients involved in this study were impaired on tests assessing knowledge of objects and on the demonstration of their use. The patients' success in object use was significantly correlated with their knowledge about the objects, providing further evidence that conceptual knowledge plays a key role in object use. Having a recipient present improved performance in the moderately impaired patients, suggesting that a certain level of conceptual knowledge must remain for the additional information to be beneficial. Although overall accuracy in using the target objects was not related to our measures of affordance, the specific aspects of use afforded by the objects' structures were relatively impervious to semantic impairment, suggesting a role for affordance information when object-specific knowledge is disrupted. The patients' familiarity with the objects was an important predictor of performance. Finally, despite good performance on tests of mechanical problem solving, the patients showed very little evidence of employing these skills in their interactions with real objects.
\end{abstract}

Patients with semantic dementia (SD) form the temporal variant of frontotemporal dementia (Hodges, Patterson, Oxbury, \& Funnell, 1992; Snowden, Goulding, \& Neary, 1989). The syndrome is associated with circumscribed temporal lobe atrophy, most severely affecting the temporal pole, anteromedial and inferolateral temporal lobes, typically bilaterally but asymmetrically. The ventromedial frontal cortex (bilaterally) and the amygdaloid complex are also often affected (Mummery et al., 2000; Mummery, Patterson, Wise, Price, \& Hodges, 1999). The selective nature of the semantic deficit in these patients has been confirmed by their good performance on assessments of current day-to-day memory, short-term verbal memory, visuospatial skills, nonverbal reasoning, phonology, and syntax until very late in the course of the disease (Hodges et al., 1992; Warrington, 1975). More recent studies have highlighted the patients' progressive loss of knowledge about the meanings of nonverbal as well as verbal stimuli, including knowledge of objects (Bozeat, Lambon Ralph, Garrard, Patterson, \& Hodges, 2000;

Correspondence concerning this article should be addressed to S. Bozeat, MRC Cognition and Brain Sciences Unit, 15 Chaucer Road, Cambridge CB2 2EF, England (e-mail: sasha.bozeat@mrc-cbu.cam. ac.uk).
Hodges, Bozeat, Lambon Ralph, Patterson, \& Spatt, 2000; Lambon Ralph \& Howard, 2000).

The syndrome of SD provides an ideal testing ground for investigating the role of conceptual knowledge in various cognitive domains. These include the issue of whether the ability to use familiar objects is reliant, in whole or in part, upon intact conceptual knowledge about those items. There have been reports of dissociations between conceptual knowledge and object use, and this has been taken as evidence for the existence of a separate action semantic system in which knowledge about the use of objects is stored independently of other forms of knowledge. This evidence will be evaluated in the Discussion section.

In the few studies thus far of object use in SD, the majority of patients have shown object use that is impaired to an extent consonant with their conceptual knowledge for the same items. Although these reports highlight the critical and central role of conceptual knowledge, there are a number of other factors that might be implicated in object use. In the recent study by Hodges et al. (2000), for example, there were a small number of instances in which the patients appeared to use an object more successfully than would be predicted by their knowledge about it. We suggested that several other factors might be influential—namely, residual conceptual knowledge (a 
relative preservation of general conceptual knowledge, such as the superordinate category), familiarity, personally relevant schemata (personal familiarity) and contexts, object affordances, and mechanical problem-solving strategies. As well as having the goal of confirming that use is directly tied to the degree of semantic impairment for the target object, the present study was specifically designed to address the role of some of these additional factors.

\section{Object Affordance}

In 1977, Gibson proposed the theory of affordances, in which he stated that information available more or less directly from the perception of an object gives clues as to its function and possible manipulations (Gibson, 1977). The search for the origins of the concept of affordance, however, takes us back to the 1930s and the work of Gestalt psychologist Koffka (1935), who proposed that the meaning or value of an object was perceived as readily as its physical features. The term valence was later coined to refer to this idea. There was one crucial difference between the concepts of affordance and valence: Whereas the valence of an object was thought to be bestowed upon it by the need of the observer, an affordance does not change with the need of the observer; it is always there to be perceived. Following Gibson, the notion of affordance was refined by Neisser (1994), who proposed that the term should be limited to the notion of physical affordances and not be in any way dependent on stored knowledge about objects. In recent studies, the term affordance has been used to refer to two potentially separate mechanisms that support object use directly from visual and/or tactile input. One is problem solving, or reasoning about the use of an object on the basis of its physical characteristics, which will be discussed below. The other is more like the Gibsonian notion of affordance, in which clues to the hold, orientation, movement, and purpose of an object are systematically related to its physical structure (e.g., if it has a sharp edge, it is used for cutting).

In neuropsychologicalcontexts, affordance has typically been characterized in a post hoc fashion: If a patient's use of an object is better than would be expected, it is assumed that the physical properties are guiding the patient toward the correct manipulation. For example, Hodges et al. (2000) reported that the SD patients did use two objects (a pair of scissors and a watering can) better than would be expected on the basis of their scant remaining conceptual knowledge for these items and suggested that the physical properties of the objects were influencing their use. The construction of a pair of scissors, for example, is such that it is almost impossible not to demonstrate the correct cutting movement. In the present study, a feature database was created, not only to improve scoring of object use, but also to identify the systematic relationships between physical features and the way an object is used. In this way, we hoped to be able to specify affordances a priori, before assessing their influence on the performance of the SD cases.

\section{Mechanical Problem Solving}

Patients with SD retain good problem-solving skills, as demonstrated, for example, by excellent performance on the Novel Tools test (Goldenberg \& Hagmann, 1998) until late in the course of the disease (Hodges et al., 2000; Hodges, Spatt, \& Patterson, 1999). It is perhaps surprising, then, that Hodges et al. (2000) observed little evidence of trial-and-error explorations by the SD patients that might have helped them to work out the correct use of real objects. The authors proposed two additional considerations that may explain this finding. First, in order to solve a problem effectively, it is necessary to know the target or goal. The goal is provided by the examiner in tests of mechanical problem solving, whereas in the demonstration of single-object use the patients must conceive the goal for themselves. The ability to deduce the correct goal for an object may be dependent on conceptual knowledge (i.e., the patient must know what the object's canonical function is). Second, the ability to work out a plausible function for an object often requires knowledge about its properties (e.g., knowledge about the materials it is made from), which may also be impaired in these patients. A further possible factor is that the mechanicalproblem-solving test used in the previous study, the Novel Tool test (Goldenberg \& Hagmann, 1998), can be accomplished purely through visual matching of the tools and the blocks, making it a nonstringent measure of real problem solving.

Several aspects of the present study were designed in order to explore these possible explanations for the apparent absence of problem-solving behavior in real object use. The patients were assessed on single-object use with and without a recipient (see below), to investigate whether the presence of the recipient provides clues as to the goal (the object's function), thereby enabling better problem solving. We also included a more difficult assessment of mechanical problem solving: mechanical puzzles (based on those designed by Ochipa, Rothi, \& Heilman, 1992), which cannot be accomplished simply on the basis of visual matching.

\section{Presence of a Recipient}

Previous studies by our group (Hodges et al., 2000; Hodges et al., 1999) have assessed use of single objects, in isolation, to enable investigation of the role of conceptual knowledge without contamination by other factors that are involved in object use. Obviously, this does not accurately reflect our everyday interaction with objects, where we would typically use two or more objects together to carry out a task. Indeed, this may go some way toward explaining how the SD patient reported by Lauro-Grotto, Piccini, and Shallice (1997) was able to prepare and cook a variety of different foods despite performing poorly on tests assessing conceptual knowledge of the same items. We propose that the recipient may benefit the patients in two ways: First, it may provide a level of context and, therefore, help to constrain the conceptual knowledge that underpins cor- 
Table 1

Demographic and Imaging Data

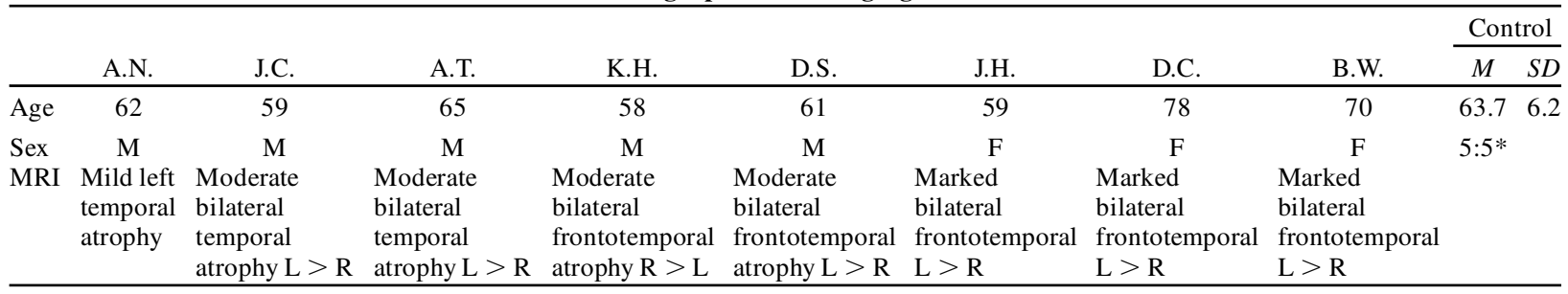

*Ratio of male to female.

rect object use; second, as has already been noted, it may provide a goal for the use of the object and, therefore, encourage problem solving.

\section{Familiarity}

Premorbid familiarity with a concept is an important predictor of many aspects of performance in SD (Bozeat et al., 2000; Funnell, 1995; Lambon Ralph, Graham, Ellis, \& Hodges, 1998). Previous assessments of object use have typically involved a corpus of highly familiar objects. In this present study, however, items were selected to cover a range of familiarity, in order to explore its influence on object use.

\section{Personally Relevant Schemata and Contexts}

Although this factor is not explored in the present study, it should be noted that repeated experience with personally familiar objects seems to help to maintain appropriate responses to them in the face of severe degradation of conceptual knowledge. From clinical reports, it is clear that patients with SD manage well with everyday tasks and often continue with hobbies until late in the course of the disease (Graham, Lambon Ralph, \& Hodges, 1997; Lauro-Grotto et al., 1997; Snowden, Griffiths, \& Neary, 1994). Two recent studies addressed this issue directly (Bozeat, Lamben Ralph, Patterson, \& Hodges, 2002; Funnell, 2001). In both, it was found that SD patients were more accurate when demonstrating the use of their own objects than with equally good substitute exemplars.

\section{METHOD}

\section{Patients}

Eight patients were identified through the Memory and Cognitive Disorders Clinic at Addenbrooke's Hospital, Cambridge, England, where they were seen by a senior neurologist (J.R.H.), a senior psychiatrist, and a clinical neuropsychologist. In addition to a clinical assessment, all the patients were given a number of standard psychiatric rating scales in order to exclude major functional psychiatric disorders, such as depression and schizophrenia. They all underwent MRI scanning, together with the usual battery of screening blood tests, in order to exclude treatable causes of dementia.

All the patients presented with a progressive loss of vocabulary that affected expressive and receptive language in the context of fluent speech production. They all fulfilled previously proposed criteria for SD: anomia, impairment in single-word comprehension, and impoverished semantic knowledge with relative preservation of phonology, syntax, visuospatial abilities, and day-to-day memory (Hodges, Graham, \& Patterson, 1995; Hodges et al., 1992). In all 8 cases, structural brain imaging by MRI showed focal atrophy involving the polar and inferolateral regions of the temporal lobes, on the left side only for the mildest case (A.N.), but bilateral in all of the others (see Table 1). In 6 of the 7 bilateral cases, there was more extensive atrophy in the left than in the right temporal lobe ( $\mathrm{L}>\mathrm{R}$ in Table 1), K.H. being the one exception $(\mathrm{R}>\mathrm{L}$ ). In the majority of cases (except for the 3 milder ones-A.N., J.C., and A.T.), the atrophy had spread from the temporal pole anteriorly to the ventromedial frontal region, as is typical in SD (Mummery et al., 2000; Snowden, Neary, \& Mann, 1996).

Ten normal subjects from the Medical Research Council Cognition and Brain Sciences Unit's subjects' panel, approximately matched in age and education to the patients, served as control subjects.

\section{General Neuropsychology}

The following battery of neuropsychological tests was administered: the Mini-Mental State Examination as a general measure of cognitive impairment (Folstein, Folstein, \& McHugh, 1975); the digit span subtest of the Wechsler Memory Scale-Revised (WMS-R; Wechsler, 1981) to assess auditory-verbal short-term memory; verbal fluency for the letters F, A, and S to test executive function; the Raven's Colored Progressive Matrices to assess nonverbal problem solving (Raven, 1962); copy and immediate recall of the Rey Complex Figure to test visuospatial skills and episodic memory (Rey, 1941). Various subtests from the Visual Object and Space Perception battery were also used to assess visuospatial function in more detail (Warrington \& James, 1986).

\section{Semantic Assessments}

The patients were given a selection of tasks from a semantic battery, which is a collection of tests that use the same set of stimulus items to assess semantic knowledge systematically across different input and output modalities. It contains 64 items representing three categories of living things (animals, birds, and fruit) and three categories of artifacts (household items, tools, and vehicles). The following subtests from the semantic battery were administered: category fluency, in which the subject is asked to produce as many exemplars as possible in $1 \mathrm{~min}$ for each of the six categories; naming of the 64 line drawings; spoken word-to-picture matching using picture arrays containing the target plus nine within-category foils. In addition, the Pyramid and Palm Trees test of associative semantic knowledge (Howard \& Patterson, 1992) was also administered. In this assessment, the subjects are asked to choose one of two items that is most closely associated with the target (e.g., for the target pyramid, the choice is between palm tree and pine tree). The stimuli are presented as either pictures or written words.

\section{General Praxis Testing}

The subjects were asked to copy the examiner in producing 10 meaningless gestures (Goldenberg, 1996). Two points were awarded for a correct gesture on the first attempt, and a single point if it was correct on the second presentation. 


\section{Mechanical Problem Solving}

1. The Novel Tools test (Goldenberg \& Hagmann, 1998). The materials for this test consist of six wooden cylinders, each of which can sit in a wooden base, and a selection of novel tools. Each cylinder has a part to which one of the tools can be fitted to lift the cylinder out of its base. During testing, one cylinder at a time is placed in the well of the base, and a collection of three tools is placed beside it. The subject is asked to select the tool best suited to lift out the cylinder. If the correct tool is not chosen as the initial response, the subject is asked to choose an alternative.

Two aspects of this task were scored separately: the selection and the use of the correct tool. For the first part, two points were given when the correct tool was selected at first choice, and one point was given if the subject selected the correct tool on the second choice (maximum score $=12$ ). The second part of the test evaluated the use of the tool (either selected by the subject or given by the examiner following two incorrect selections). Two points were awarded if the subject inserted the tool and lifted the cylinder without hesitation or error, and one point was awarded if the subject demonstrated the correct use after trial and error (maximum score $=12$ ).

2. Mechanical Puzzles (Ochipa et al., 1992). The stimuli consisted of nine clear, Perspex cylinders, each containing a wooden block and a selection of novel tools. The goal was always to remove the block from the cylinder, and each task required both the selection of the appropriate tool and the use of a different procedure/strategy (e.g., lifting, levering, pushing, pulling, etc.). During testing, one cylinder at a time was placed in front of the subject, and a collection of four tools was placed beside it. The subject was asked to select the tool best suited to remove the block out of the cylinder.

Once again, the two aspects of this task were scored separately. For the first part, one point was given when the correct tool was selected at first choice (maximum score $=9$ ). To evaluate use of the tool (either selected by the subject or given by the examiner following an incorrect selection), two points were awarded if the subject inserted the tool and removed the block without hesitation or error, and one point was awarded if the subject demonstrated the correct use after trial and error (maximum score $=18$ ).

\section{Object Use Battery}

A multiple component battery was constructed with the purpose of assessing associative information, functional knowledge, and use of 36 household objects. These were derived from three categoriestools, kitchen implements, and stationery items - and covered a fairly wide range of rated familiarity.

Tests of conceptual knowledge. Conceptual knowledge for the 36 objects was assessed in a series of matching tests, which consisted of digital photographs of the targets and similar photographs of four possible matches for each target. The picture of the target object was located at the top of the page, and the subject was asked to choose one of the four response alternatives as the best match according to one of three types of relationship, described below. The order of items was randomized across tasks, and each was preceded by four practice trials.

Every effort was made to ensure comprehension of the task. Data were not included if there was any doubt about the patients' ability to comprehend the instructions, which occurred in 3 subjects (J.H., D.C., and B.W.) when they were asked to match according to the action that would be used when the target object was manipulated.

1A. Matching to Recipient. The subjects were asked to choose the correct typical recipient for the target object. The foils were chosen to be visually similar to the correct match or semantically related (e.g., for the target garlic press, the recipient choice is between garlic, onion, pepper, and cheese).

1B. Matching to Function. In this test, the subjects were asked to choose one of four objects that could be used for the same purpose as the target item. The foils were chosen to be either visually similar to or from the same category as the target (e.g., for the target gar- lic press, the choice is between pestle and mortar, corkscrew, scissors, and pliers).

1C. Matching to Action. In this test, the subjects were asked to choose one of four objects that would be manipulated /moved in the same way as the target. The object that represented the correct choice is not necessarily held in the same way as the target but requires a similar action. The foils were chosen to be visually similar or semantically related to the target (e.g., for the target garlic press, the choice is between secateurs, corkscrew, bottle opener, and compasses).

2. Naming. The subjects were given each object individually and were asked to produce the name.

3. Word-to-picture matching. The subject was asked to choose the item from picture arrays containing the target plus seven withincategory foils, in response to the spoken target name.

4. Action-to-picture matching. In this test, use of the object was mimed by the experimenter, and the subject was asked to choose the object being used from an array of eight within-category items. These were the same arrays as those used in the word-to-picture matching test. Data were not included for one patient, D.C., who was unable to comprehend this task.

5A. Single-object use. The subjects were given each real object in isolation and were asked to demonstrate its use. Performance was videotaped for later evaluation.

$5 B$. Object use with recipient. Only a subset of the 36 items were tested with a recipient $(n=22)$, because some were not practical (e.g., potato masher) and others did not have a typical recipient (e.g., tape measure). The subjects were given each object and its recipient and were asked to demonstrate their use together. Performance was videotaped for later evaluation.

\section{Scoring Object Use}

A feature database was constructed to enable the quantification of the physical affordances of the objects (details below) and also to create a feature-based scoring scheme in place of the rater-based methods used in many other studies of object use. Object use by the control subjects was examined first, and from these data, a template was formed for each object. This template specified a description of the canonical use of each object in terms of composite features: the number of hands used to hold the object, the grasp, the position on the object, and each individual movement. So, for example, the featural description for the use of a hammer was the following: held in one hand, with a "standard" grasp, about half-way down the handle, with the flat end of the head facing down; lift-up, strike down; movement repeated. The control subjects' and the patients' object use videos were then scored according to this template, leading to separate scores for the hold, movement, and orientation of the object. Because the total possible score for the hold and movement varied across objects, proportional scores were used in all the analyses.

\section{RESULTS}

\section{General Neuropsychology}

The 8 patients covered a broad spectrum of impairment, as is indicated by their performance on the MiniMental State Examination (see Table 2, in which the patients are ordered by their overall performance on the three object-matching tasks). Five of the 8 cases showed intact working memory as measured by forward and backward digit span. D.C.'s forward span was (just) within normal limits, but she could not comprehend the instructions for backward span. D.S. and B.W., who, like D.C., had a profound anomic aphasia, were also slightly subnormal even on forward verbal span. All the patients except A.N. ex- 
Table 2

General Neuropsychology

\begin{tabular}{|c|c|c|c|c|c|c|c|c|c|c|}
\hline \multirow[b]{2}{*}{ Test (Maximum Score) } & \multicolumn{8}{|c|}{ Subject } & \multicolumn{2}{|c|}{ Control } \\
\hline & A.N. & J.C. & A.T. & K.H. & D.S. & J.H. & D.C. & B.W. & $M$ & $S D$ \\
\hline MMSE (30) & 30 & 24 & 25 & 22 & 12 & 7 & 8 & 7 & 28.8 & 0.5 \\
\hline \multicolumn{11}{|l|}{ Digit span } \\
\hline Forward & 7 & 7 & 8 & 6 & 4 & 6 & 5 & 4 & 6.8 & 0.9 \\
\hline Backward & 7 & 4 & 5 & 5 & 4 & 5 & 0 & 3 & 4.7 & 1.2 \\
\hline Letter fluency (total: FAS) & 40 & 22 & 20 & 13 & 2 & 8 & 0 & 0 & 44.2 & 11.2 \\
\hline Raven's colored matrices* & 95 & 75 & $90-95$ & 95 & $75-90$ & 95 & 95 & 75 & & \\
\hline \multicolumn{11}{|l|}{ VOSP } \\
\hline Incomplete letters (20) & 20 & 18 & 20 & 20 & 20 & 17 & NT & 19 & 19.2 & 0.8 \\
\hline Dot counting (10) & 10 & 9 & 10 & 10 & 10 & 10 & 10 & 10 & 9.9 & 0.3 \\
\hline Position discrimination (20) & 20 & 19 & 19 & 20 & 20 & 19 & NT & NT & 19.8 & 0.6 \\
\hline Cube analysis (10) & 10 & 10 & 10 & 10 & 10 & 10 & 10 & 9 & 9.7 & 2.5 \\
\hline Number location (10) & 10 & 9 & 10 & 10 & 10 & 10 & NT & NT & 8.9 & 2.8 \\
\hline
\end{tabular}

Note-Patients are ordered according to their performance on the object matching tests. VOSP, Visual Object and Space Perception battery; NT, not tested. *Scores on the Raven's matrices are given as percentiles.

hibited some impairment on the letter fluency test, with the three most anomic cases being outliers. There was general preservation of nonverbal problem-solving and visuospatial skills, as measured by Raven's Colored Progressive Matrices and the Visual Object and Space Perception battery.

\section{Semantic Assessments}

As is shown in Table 3, the patients included in this study covered a wide range of semantic decline, from the very mildly impaired patient A.N., whose deficits were only revealed by a subset of the more taxing assessments, to B.W., who had profound semantic degradation. All the patients except A.N. showed reduced category fluency, some degree of anomia as indicated by their performance on the naming test, and impaired comprehension as measured by the word-to-picture matching (note that the patients in Tables 2 and 3 are ordered as best as possible to reflect decreasing scores on the three object-matching tests). A number of patients with semantic deficits, especially from herpes simplex virus encephalitis, have been reported in the literature to show dissociations in performance according to category of knowledge (e.g., living vs. manmade). Looking at the performance on the word-to-picturematching test, all but one of the impaired patients had nu- merically better scores on items from the artifact domain, although this difference was very small in most cases. One patient, D.S., performed considerably better on the manmade items (25/32 vs. $15 / 32)$, but it should be noted that his performance on items from both domains is well below that of the control subjects. All the patients were impaired on both conditions of the Pyramid and Palm Trees test, except for A.N., whose performance was normal on the picture version.

\section{General Praxis Testing}

An independent samples $t$ test revealed no difference between the performances of the patients and the control subjects on copying the meaningless gestures $[t(16)=$ 1.57 , n.s.], indicating that the patients suffered from no significant impairments to general praxis.

\section{Mechanical Problem Solving}

The patients performed well on both selection and use of the tools in both mechanical-problem-solving tasks (see Figure 1). A repeated measures analysis of variance (ANOVA) confirmed that there was no reliable difference between the performances of the patients and the controls $[F(1,16)=3.14, p>.05]$. There was a significant effect of task component $[F(3,48)=19.89, p<.001]$, with se-

Table 3

Assessment of Semantics

\begin{tabular}{|c|c|c|c|c|c|c|c|c|c|c|}
\hline \multirow[b]{2}{*}{ Test (Maximum Score) } & \multicolumn{8}{|c|}{ Subject } & \multicolumn{2}{|c|}{ Control } \\
\hline & A.N. & J.C. & A.T. & K.H. & D.S. & J.H. & D.C. & B.W. & $M$ & $S D$ \\
\hline \multicolumn{11}{|l|}{ Category fluency } \\
\hline Living & 47 & 17 & 14 & 8 & 0 & 5 & 0 & 0 & 60.3 & 12.6 \\
\hline Manmade & 34 & 23 & 18 & 14 & 0 & 7 & 0 & 0 & 54.8 & 10.3 \\
\hline Naming (64) & 64 & 41 & 17 & 42 & 1 & 6 & 2 & 1 & 62.3 & 1.6 \\
\hline Word-picture matching (64) & 64 & 56 & 57 & 51 & 40 & 18 & 23 & 8 & 63.7 & 0.5 \\
\hline Living & 32 & 25 & 27 & 23 & 15 & 10 & 9 & 3 & 31.8 & 0.4 \\
\hline Manmade & 32 & 31 & 30 & 28 & 25 & 8 & 14 & 5 & 32.0 & 0.2 \\
\hline \multicolumn{11}{|l|}{ Pyramids and Palm Trees } \\
\hline Words (52) & 48 & 44 & 45 & 40 & 40 & 25 & NT & NT & 51.1 & 1.1 \\
\hline Pictures (52) & 51 & 41 & 47 & 42 & 41 & 34 & 29 & 30 & 51.2 & 1.4 \\
\hline
\end{tabular}

Note-NT, not tested. 


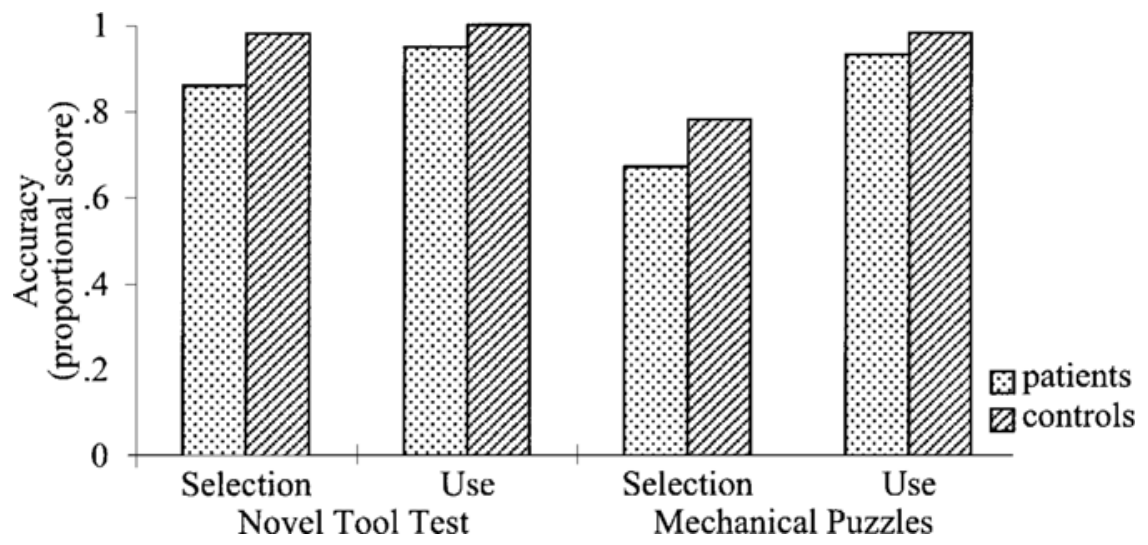

Figure 1. Performance on the Novel Tools test and mechanical puzzles.

lection (not surprisingly) proving more difficult than use, but no interaction between group and task component $[F(3,48)<1]$.

\section{Object Use Battery}

Object matching tests. Every patient was impaired on all three matching subtests, except for A.N., who performed within the normal range on one of the three, matching to recipient (see Figure 2, in which the patients are ordered by their performance on these three tasks). The three most impaired patients do not have scores for the matching-to-action subtest, because they were unable to comprehend the instructions for this component. A repeated measures ANOVA revealed a significant main effect of group [controls $>$ patients, $F(1,13)=52.2, p<$ $.001]$ and test $[F(2,26)=10.9, p<.001]$, but no inter- action between these two factors $[F(3,39)=1.5, p>.05]$. Post hoc tests confirmed that the patients' scores on all three matching tests were significantly lower than the scores of the control subjects $(t$ values between 4.9 and 7.2 , all $p \mathrm{~s}<.01)$. Numerically speaking, the patients performed best on matching to recipient and most poorly on matching to function. Perhaps owing to the high variability within the group, none of the differences between these three tests was statistically significant ( $t$ values between 0.48 and 1.08 , n.s.). In terms of a different criterion (the ability to comprehend and, therefore, complete the task), performance was worst on matching to action.

The control subjects performed better on matching to recipient than on either of the other two matching tests ( $t$ values of 2.7 and 4.5 , both $p$ s $<.05$ ), and their scores on matching to function were significantly higher than

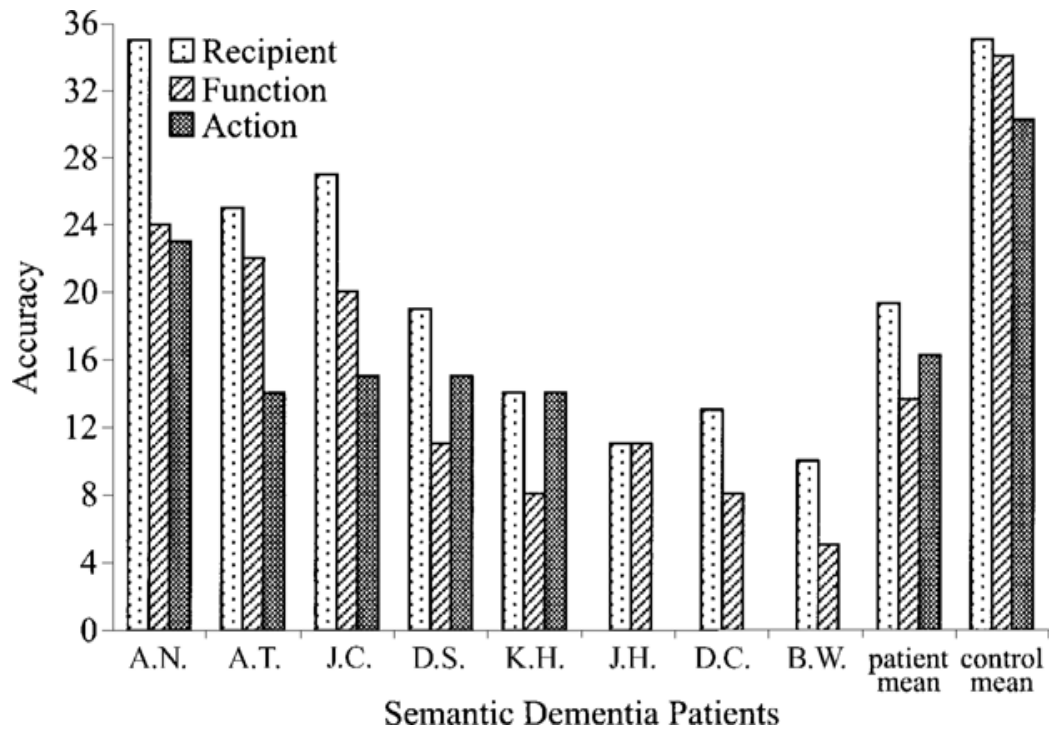

Figure 2. Performance on the three tests of visual associative knowledge. Data are not included for three patients, J.H., D.C., and B.W., on the action-matching test because they were unable to comprehend the task. 


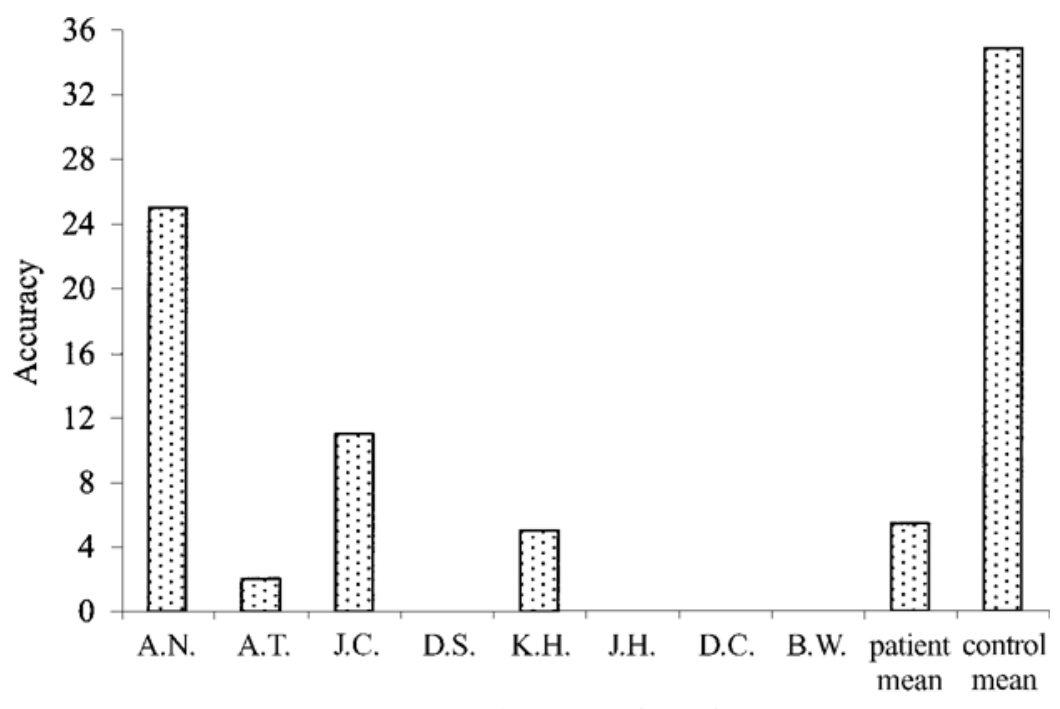

Semantic Dementia Patients

Figure 3. Performance on naming the objects.

their scores on matching to action $[t(9)=3.4, p<.01]$.

Sirigu, Duhamel, and Poncet (1991) reported a patient who was unable to recognize the function of objects but was, nevertheless, often able to demonstrate appropriate manipulations (this case will be considered in further detail in the Discussion section). The opposite dissociation, preserved function knowledge and impaired manipulation knowledge, was reported in two cases by Buxbaum, Veramonti, and Schwartz (2000). In the present data, there was no significant difference between the performance of the patients as a group on matching to function versus matching to action, although the performance of the control subjects indicated that the action task was more difficult. As individuals (see Figure 2), 2 of the patients (D.S. and K.H.) showed a reversal of the relative difficulty in these two subtests, relative to the control subjects (i.e., better performance on matching to action than on matching to function), but this difference was not significant in either case (both $x^{2}<1$, n.s.).

Naming. The patients were impaired, all except A.N. profoundly so, at naming the objects (see Figure 3). Four patients (D.S., D.C., J.H., and B.W.) failed to name any of the 36 objects. A $t$ test confirmed that the patients' scores on naming were significantly lower than those of the control subjects $[t(16)=9.38, p<.001]$.

Word-to-picture matching and action-to-picture matching. It is clear from Figure 4 that all the patients were impaired at selecting the objects in response to both their spoken names and a pantomime of their use. It was confirmed with $t$ tests that the patients' scores on both these tasks were significantly lower than the scores of the control subjects' $[t(16)=4.92, p<.01 ; t(15)=6.6, p<$ $.001]$. There was no difference between the patients' performances on these two tasks $[t(6)<1]$.

\section{Object Use}

Figure 5 reveals that the patients' ability to demonstrate the correct use of the objects was poorer than that of the control subjects on all three dimensions (hold, movement, and orientation). A repeated measures ANOVA revealed significant main effects of group $[F(1,16)=34$. , $p<.001]$ and object use component $[F(2,32)=31.1$, $p<.001]$, plus an interaction between these two factors $[F(2,32)=14.1, p<.001]$. The scores of the patients were significantly higher on correct hold for the objects than on either movement $[t(7)=5.68, p<.05]$ or orientation $[t(7)=2.64, p<.05]$ and were significantly higher on orientation than on movement $[t(7)=3.53, p<$ $.05]$. The control subjects scored best on the orientation component, with scores on this component being slightly but, nevertheless, significantly higher than scores on the hold $[t(9)=2.51, p<.05]$ and the movement of the objects $[t(9)=5.06, p<.001]$. Like the patients, the control subjects' scores on correct hold were significantly higher than those on movement $[t(9)=6.01, p<.001]$.

\section{The Relationship Between \\ Conceptual Knowledge and Object Use}

Pearson's correlations revealed significant associations between virtually all combinations of the patients' scores on the various semantic tests: the five from the semantic battery (category fluency, picture naming, word-to-picture matching, and the word and picture conditions of the Pyramid and Palm Trees test) and the two designed for this study (naming the objects, and a combined score for the three associative matching tasks; $.63<r<.95$, all $\left.p \mathrm{~s}_{\text {one-tailed }}<.05\right)$. The only exceptions were the correlations between the word condition of the Pyramid and Palm Trees test and two naming tests, which failed to reach con- 


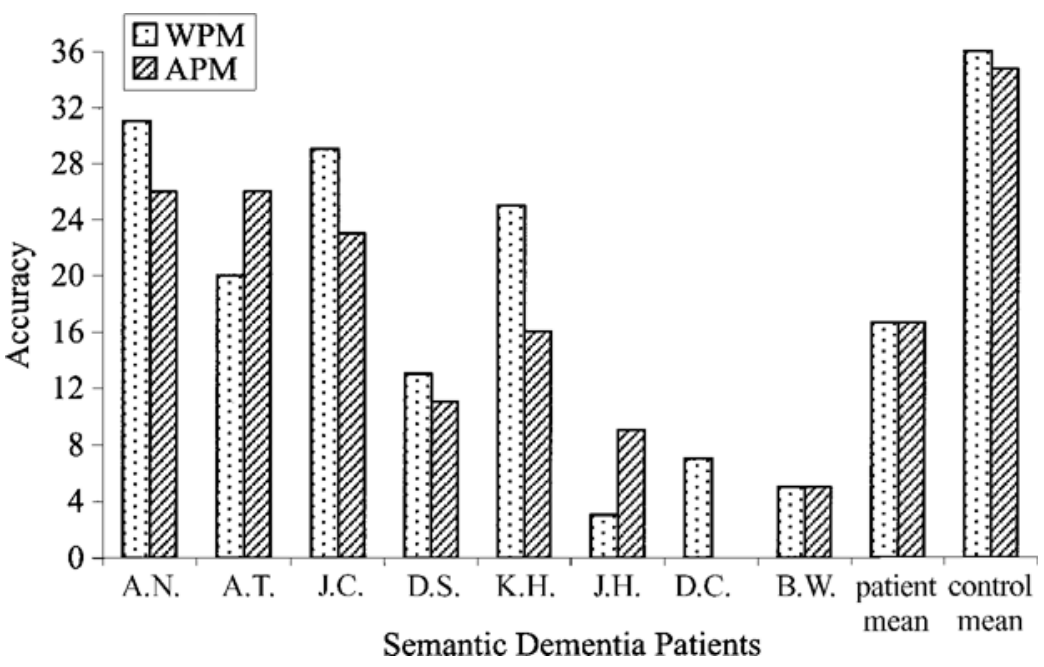

Figure 4. Performance on the word-to-picture matching (WPM) and action-topicture matching (APM) tests. Data are not included for D.C. on APM because she was unable to comprehend the task.

ventional levels of significance $(r=.62, p=.09 ; r=.61$, $p=.010$ ), perhaps because 2 of the patients were not tested on the word condition of the Pyramid and Palm Trees test. These correlations support the view that the impairments in these patients reflect damage to a central, amodal system that underpins conceptual knowledge (Bozeat et al., 2000; Lambon Ralph \& Howard, 2000).

One of the aims of this study was to replicate the results of the previous study reported by Hodges et al. (2000)that is, to demonstrate the importance of conceptual knowledge in object use. In keeping with this hypothesis, both overall use and each of the individual components of object use (hold, movement, and orientation) correlated re- liably with all of the semantic tests designed for this study and with virtually all of the semantic assessments reported in Table $3\left(.68<r<.91\right.$, all $\left.p \mathrm{~s}_{\text {one-tailed }}<.05\right)$. Only correlations of the word condition of the Pyramid and Palm Trees test with movement and overall use failed to reach conventional levels of significance $(r=$ $.64, p=.08 ; r=.67, p=.07)$.

By-subjects regression analyses were carried out to determine whether any individual patient's performance was discrepant from the significant group-based relationship between object use (the total score on the three components) and knowledge (as measured by the total score on the three associative matching tests and word-to-picture

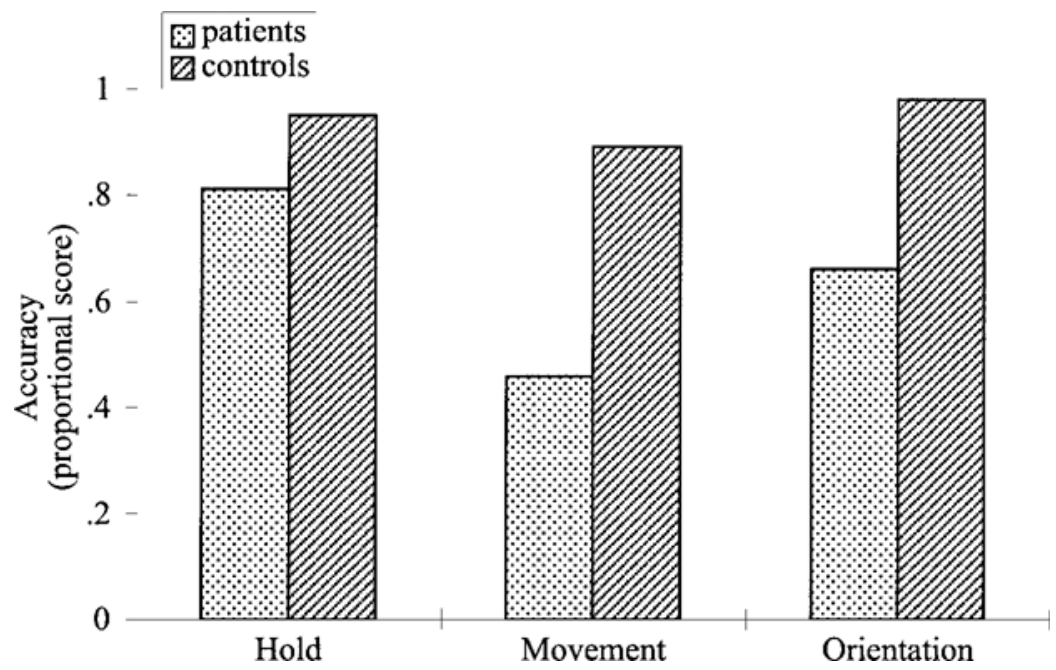

Figure 5. Performance on the individual components of object use. 


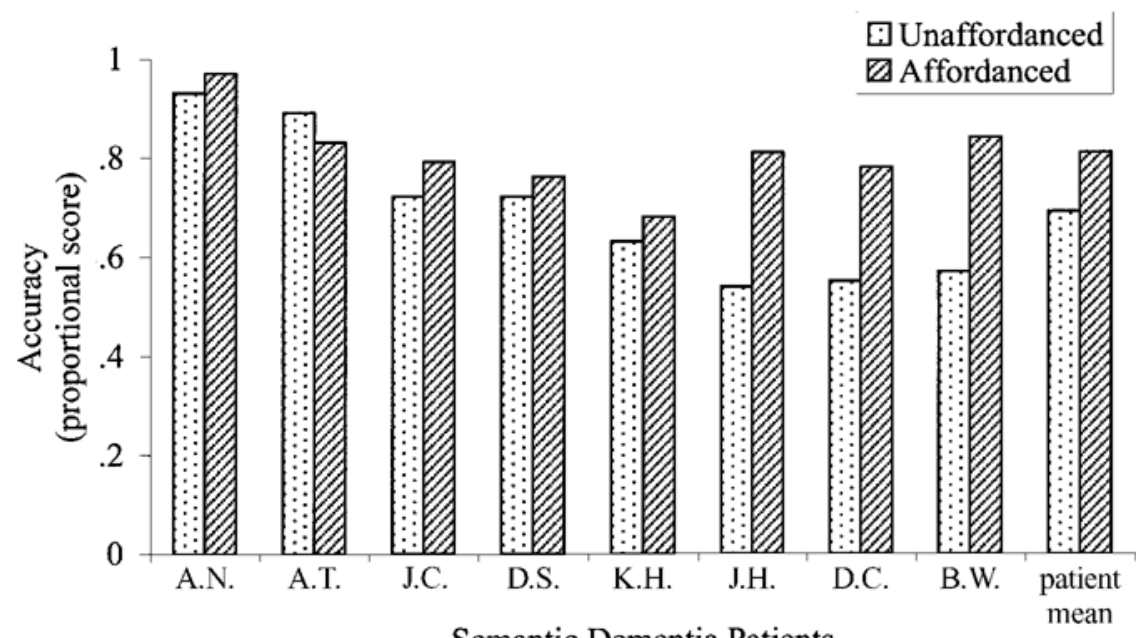

Semantic Dementia Patients

Figure 6. Performance on affordanced and unaffordanced objects. In some objects, the hold is afforded, whereas in others, it is the movement. In all cases, however, the score depicted is for overall use.

matching). With two standard residuals either side set as the criterion, none of the 8 patients deviated significantly from this relationship.

\section{Affordances}

Specifying affordances empirically. Gibson's theory of affordances proposes that information available directly from perception gives clues as to the function of an object and the possible manipulations of it (Gibson, 1977). A feature database was constructed to enable a priori quantification of these affordances. The database contained 90 manmade objects, and each one was rated according to a large number of structural features $(n=56)$, including overall size, the number of handles, the type of handle(s), the position of the handle in relation to the end of the tool, the presence of moving parts, and what was at the end of the tool. Various features of the hold ( $n=11$; e.g., the number of hands, position on the tool, grasp), of each individual movement ( $n=17$; e.g., lift up, strike down), and of the function of each object $(n=21$; e.g., cutting, cleaning) were also specified. Systematic relationships between features were highlighted by computing Spearman's correlations for each possible pairing across the 90 objects. In total, there were 105 features, which led to 5,460 possible feature-feature pairings; it was surprising, therefore, to find only 46 significant correlations.

It is important to consider the chance level when performing such a large number of correlations: In this case, one would expect 273 significant correlations to occur by chance. The number of observed reliable correlations was, therefore, significantly lower than would be expected by chance $(z=-14.10, p<.001)$.

The significant correlations obtained can be classified in the following ways: (1) structural feature-structural feature ( $n=11$; e.g., if the object has two handles, it is likely to have moving parts); (2) structural feature-hold ( $n=8$; e.g., if the object has a handle that joins the shaft, it is likely to be held in a "standard" grasp); (3) structural feature-movement ( $n=5$; e.g., if the object has a wedgeshaped head, it is likely to be associated with a striking down movement); (4) structural feature-function ( $n=11$; e.g., if the object has a sharp, serrated edge, it is likely to be used for cutting); (5) hold-hold ( $n=2$; e.g., if one hand is a "pinch" grip, the other hand is likely to be a "pinch" grip as well); (6) hold-movement ( $n=1$; e.g., if the second hand is a "pinch" grip, it is likely to be twisted horizontally with the fingers); (7) movement-movement ( $n=5$; e.g., if the object is "lifted-up." it is likely to be associated with a "striking-down" movement as well); (8) movement-function $(n=3$; e.g., if the object is held still, it is likely to be used for measuring).

Do affordances influence object use? Twelve affordanced objects were selected on the basis that either the hold or the movement was reliably predicted by a structural characteristic in the analysis of the feature database described above. These were matched on the basis of familiarity to 12 other objects for which neither the hold nor the movement was obviously afforded by their structure. Performances on the affordanced and unaffordanced set were compared both for overall use score and on the particular target component of use (hold or movement). No difference was revealed by $t$ tests on scores of overall use $[t(11)<1]$ or of the particular component that was afforded $[t(11)=$ $1.58, p>.05]$. It is clear from Figure 6, however, that object use by some of the patients benefited from these affordances, and the level of semantic impairment appears to be an important factor. When the patients were subdivided into two groups according to their level of semantic impairment, the more impaired patients $(n=4)$ achieved significantly better performance on the particular component of 


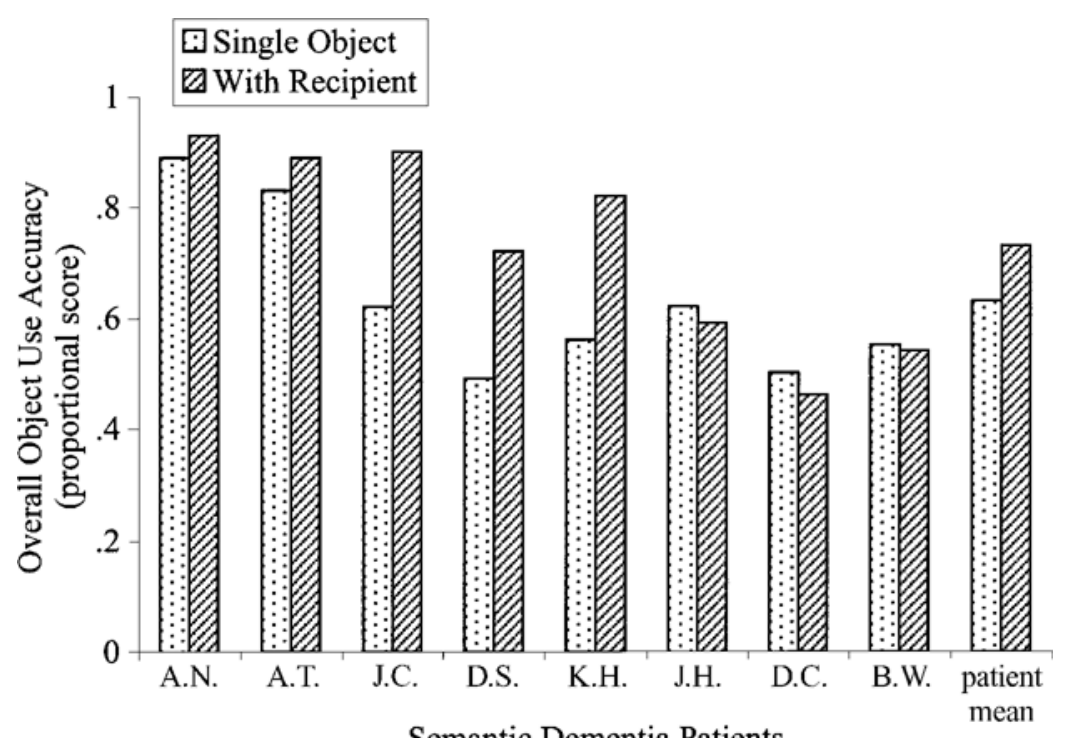

Figure 7. Performance on single-object use and use with recipient.

use that was afforded, as compared with objects with no such specific affordances $[F(1,3)=13.6, p<.05]$, whereas the mildly impaired patients showed no difference $[F(1,3)<1$, $n=4]$. In the most impaired patients (J.H., D.C., and B.W.), there was also an interaction between affordance and familiarity $[F(1,2)=32.6, p<.05]$, suggesting that familiarity is only important in the use of unaffordanced items; for affordanced objects, there was no influence of familiarity.

The difference between component use scores on the affordanced and unaffordanced items correlated significantly with overall semantic knowledge scores $(r=-.86$, $p<.01)$. This demonstrates in a different way that the level of semantic impairment is a critical factor in determining the impact of affordances on object use.

\section{Presence of Recipient}

Our everyday interaction with objects typically involves using pairs of objects together (one object and its recipient) to complete a task (e.g., using a hammer to drive a nail, a corkscrew to open a bottle of wine, a potato masher to mash potatoes, etc.). As was explained in the Method section, in order to explore the impact of the recipient, we reassessed use of 22 of the objects, on a different occasion, with the recipient present.

Five of the patients showed a numerical advantage for overall object use with the recipient present. This difference was very small in the 2 patients with mild semantic impairment (A.N. and A.T.) but was quite striking in 3 patients with more moderate semantic impairment (see $\mathrm{Pa}$ tients J.C., D.S., and K.H. in Figure 7). Analysis of the patients as a group revealed that the scores on correct hold for the objects were significantly higher when the recipient was also present $[F(1,7)=10.4, p<.05]$; scores were also higher on correct movement, although this did not reach significance $[F(1,7)=4.5, p=.07]$. There was no difference between these two conditions on scores of orientation $[F(1,7)<1]$ or overall use $[F(1,7)<1]$. The patients were then subdivided into three groups according to their level of semantic impairment; a repeated measures ANOVA revealed significant effects of severity group $[F(2,5)=16.6, p<.01]$ and presence of recipient $[F(1,5)=173.8, p<.001]$ and a significant interaction between group and recipient $[F(2,5)=163.9, p<.001]$. Post hoc tests confirmed that only the moderately impaired patients $(n=3)$ scored significantly better with the recipient present $[t(2)=17.7, p<.01]$; there was no difference between performance with and without a recipient in the mildest patients $[t(1)=5, p>.05, n=2]$ or in the most impaired patients $[t(2)=3.02, p>.05, n=3]$.

\section{Familiarity}

Familiarity is an important predictor of performance on tasks assessing conceptual knowledge (Bozeat et al., 2000; Funnell, 1995; Lambon Ralph et al., 1998), so it was predicted that it would be an important factor in object use. Familiarity ratings were initially obtained by asking 20 normal, age-matched subjects to rate how often they use each object. The 36 items in the object use battery were chosen to cover a range from highly familiar items that are used by most people on a daily basis (e.g., a pencil) to less familiar items that are used by most people only about once a year (e.g., a chisel). From inspection of these ratings, it became clear that familiarity varies greatly from one person to another, being highly dependent on career and lifestyle. These ratings were used to create the battery of items, but it was decided that they would not be suitable for analysis of the effects of familiarity on the patients' object use. Most of the patients involved in this study had been suffering from SD for several years, and as a consequence, their hobbies and daily activities were 

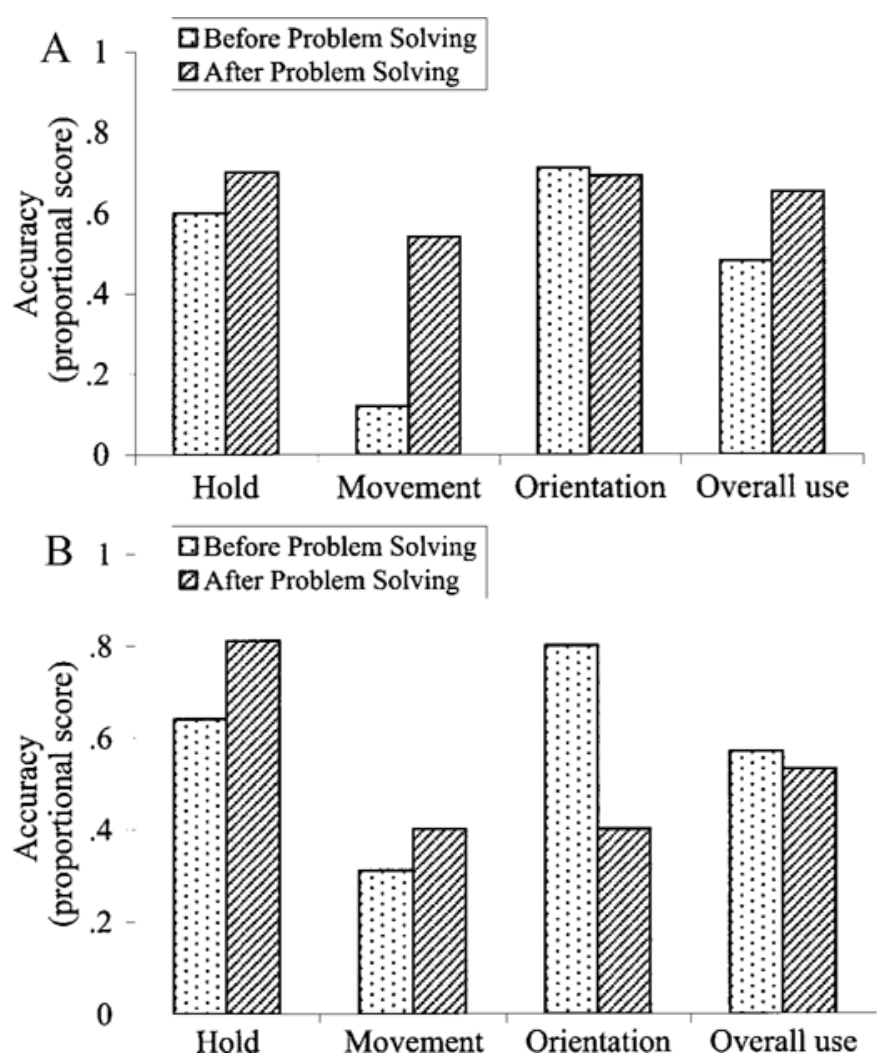

Figure 8. (A) Performance of Patient K.H. before and after problem solving. (B) Performance of Patient J.H. before and after problem solving.

greatly reduced. Ratings of familiarity were therefore obtained for each patient from his or her spouse or caregiver. Pearson's correlations revealed a significant association between the patients' success in overall object use and the familiarity ratings collected from the patient caregivers $\left(r=.39, p_{\text {one-tailed }}<.01\right)$.

Although not the main point of this analysis, it is of interest to note that the spouse/caregiver ratings indicated that the patients did indeed have much less contact with most of the objects than did the control subjects. A $t$ test confirmed that the familiarity ratings for the patients were significantly lower than the ratings obtained from the control subjects $[t(7)=11.53, p<.001]$. It is furthermore interesting to note, however, that some of the patients were assigned surprisingly high familiarity ratings with some objects that, from the ratings obtained from the control subjects, were deemed to be relatively low in familiarity. For example, D.C. was reported to use a tape measure every day to measure the length and width of jigsaw puzzle boxes in order to cut pieces of Sellotape to the exact size for fastening the boxes.

\section{Problem Solving}

All the patients performed well on the tests of mechanical problem solving, the Novel Tool test and the mechan- ical puzzles. We wanted to determine, therefore, whether they were utilizing these good problem-solving skills in their use of real objects. Because we had predicted that presence of a recipient might enhance problem-solving behavior, the first analysis compared object use with and without a recipient.

Overall, there was no significant difference in the rate of problem solving (defined by at least two attempts to use an object in different ways) between use of the objects with and without a recipient $[t(8)=2.02, p=.08]$. Only 2 patients (K.H. and J.H.) were found to use trial and error consistently across a number of items. In order to explore the impact of this problem-solving behavior in these two cases, we compared object use scores on the first attempt with those achieved on the last attempt. The analysis produced mixed results, with K.H. showing a significant improvement on one component of object use, whereas J.H. demonstrated no improvement on any of the components (see Figure 8). The score achieved by K.H. on the movement of the objects was significantly higher following problem solving $[t(13)=3.8, p<.01]$; his overall use was also better following problem solving, although this did not reach significance $[t(13)=1.9, p=$ .07]. There was no difference between scores on hold $[t(13)<1]$ or orientation $[t(13)<1]$ before and after 
problem solving. J.H. showed no improvement on overall use $[t(12)<1]$ or on any of the individual components [hold, $t(12)=1.39, p>.05$; movement, $t(12)<1$ ]; in fact, her scores on orientation were significantly higher before trial-and-error problem solving $[t(12)=2.74, p<$ .05]. These results suggest that the patients do not often make use of their good problem-solving skills to work out what to do with objects; and even when they do, it need not be beneficial for all aspects of use.

\section{DISCUSSION}

In a previous study (Hodges et al., 2000), competence in the use of familiar objects by patients with SD was significantly predicted by the patients' degree of retained/ disrupted conceptual knowledge for the same objects. The primary aims of the present study were (1) to replicate the results of the previous study, using a more comprehensive battery of conceptual knowledge tests, a larger corpus of items, and a feature-based approach to scoring object use, and (2) to investigate the influence of a range of other factors that may impact on object use, including object affordance, presence of a recipient, familiarity, and mechanicalproblem-solving strategies. The results for each of these factors is summarized and discussed, in turn, below.

\section{Conceptual Knowledge}

Seven of the 8 patients involved in this study were impaired on all the tests assessing conceptual knowledge, with one case (A.N.) showing deficits on a subset of these assessments. All the patients (again with the exception of A.N.) were impaired at demonstrating the use of the objects, and across the 8 cases, success in object use was significantly correlated with level of conceptual deficit. Taken together with the results of our previous study (Hodges et al., 2000) and those of Hamanaka and collegues (Hamanaka et al., 1996), 18 cases of SD have now been reported in which deficits in object use in line with the patients' conceptual impairment have been shown. It is also important to note that the impact of two other factorsnamely, the presence of a recipient and affordance-was modulated by the level of conceptual impairment (this finding will be discussed in further detail below). These results provide strong evidence for the key role played by conceptual knowledge in object use.

Dissociations between knowledge about an object's function and its manipulation have been reported in the literature (Buxbaum et al., 2000; Sirigu et al., 1991), and it has consequently been suggested that certain types of conceptual knowledge about objects may be more critical for their use than are others. We found no evidence for dissociations between different types of knowledge: The patients were equally impaired on all aspects of conceptual knowledge. We should emphasize, however, that this conclusion applies to the use of single objects, as was assessed here. There may be other forms of knowledge, which may be conceptual or more accurately described as proce- dural, that help to support action in naturalistic settings where the patient has (1) a goal in mind and (2) a whole relevant context in which to act on and with the object(s).

\section{Impaired Object Use in the \\ Context of Preserved Semantic Knowledge}

Several patients have been reported in the literature who were unable to use real objects correctly despite having preserved knowledge about those same objects (Rumiati, Zanini, Vorano, \& Shallice, 2001; Spatt, Bak, Bozeat, Patterson, \& Hodges, 2002). These patients invariably had some level of ideomotor apraxia associated with damage to parietal regions, which left them unable to produce the movements appropriate for object use. There has been some controversy in the literature as to whether the concept of ideomotor apraxia should be limited to tests of pantomime and imitation or whether it also has an impact on real object use. Zangwill (1960) noted that difficulties in using real objects may be related to a severe production disorder. In concordance with this, we have reported a group of patients with ideomotor apraxia owing to corticobasal degeneration, who had difficulties demonstrating the use of real objects (Spatt et al., 2002).

Ochipa, Rothi, and Heilman (1989) reported a lefthanded patient who, following a right-hemisphere stroke, was able to name objects but was unable to point to them when their functions were described or to describe their functions himself. Furthermore, he was unable to demonstrate their uses. This inability to use tools could not be explained solely by a production deficit, because he was also unable to match tools to their recipients, suggesting an impairment in the appreciation of the functional relationship between different objects. The authors proposed that this patient was suffering from an impairment in the action semantic system. Closer inspection of these data, however, suggested deficits on other semantic tasks as well. For example, the patient succeeded in naming 17/20 of the objects in the experimental battery (no control data were reported, but these objects were described as "common household tools and objects," implying that most people would perform at ceiling on this task), and he scored just $48 / 60$ on an alternative naming task. His performance was undoubtedly better on general semantic tasks than on tasks assessing knowledge of tool use, but this pattern of results is perhaps explicable in terms of task difficulty. Describing the function of objects and, indeed, selecting objects in response to descriptions of their function are more linguistically demanding tasks than simply naming objects or selecting them in response to their spoken names.

Furthermore, this patient had suffered fairly extensive brain damage (including frontal, inferior parietal, and superior temporal regions), which is likely to have affected a number of cognitive domains. Although the authors argued that the semantic impairment was confined to the action domain, it seems plausible that there was at least a degree of impairment in general semantic knowledge. Further- 
more, the patient was observed to misuse common objects in their natural settings, in a manner suggestive of a frontal dysexecutive syndrome. It is, therefore, not so clear that this patient had selective damage to an action semantic system.

Moreaud, Charnallet, and Pellat (1998) reported the same dissociation-impaired object use in the context of preserved conceptual knowledge-in a patient with moderate stage Alzheimer's disease. Despite performing well on tests tapping knowledge of 15 common household objects and preserved praxis, this patient was not always able to demonstrate their use correctly. Once again, however, careful inspection of these data revealed that the patient did not always perform well on the tasks assessing conceptual knowledge. For example, E.J. was able to provide names and describe the use of only 3 of the 7 objects that he failed to use correctly. In fact, there were only 2 objects that E.J. failed to use, despite demonstrating preserved knowledge, as assessed by all the semantic tasks. These objects were a camera and a corkscrew, which, depending on the exemplar, can be fairly complicated to use. Like the case reported by Ochipa et al. (1989), this patient was also reported to show marked difficulties with executive functioning.

\section{Preserved Object Use in the \\ Context of Degraded Semantic Knowledge}

Patients with SD seem to manage surprisingly well with everyday tasks and have been reported to use a number of objects correctly, even the same objects to which they cannot provide names, descriptions, or correct associative semantic judgments. Such observations are, however, largely anecdotal, with few investigations having systematically explored the use of real objects. Buxbaum, Schwartz, and Carew (1997) reported a patient who, despite a moderate degree of semantic impairment, used most objects normally. In this study, however, the authors did not assess knowledge about and usage of the same objects, precluding a definitive conclusion that this patient was able to use objects for which he had degraded semantic knowledge. A study by Lauro-Grotto et al. (1997) assessed the ability of another patient with SD to prepare food, which she did without error for nearly all ingredients, despite performing poorly on verbal tests assessing knowledge of the same items. This study, however, did not assess single-object use, and it is possible that the patient's successful use of kitchen tools and ingredients may have benefited strongly from the rich contextualenvironment in which she was tested.

In contrast, three studies have concluded that semantic impairment does lead to deficits in object use. Hamanaka et al. (1996) reported the co-occurrence of impoverished conceptual knowledge and impaired object use in two SD patients. There is some indication from this report that the degree of semantic impairment may be a critical factor. One of the patients initially presented with a mild semantic deficit affecting verbal comprehension and production and, at that stage, had preserved object use. Over time, however, as the patient's comprehension deteriorated further, the ability to use common objects declined too. Hodges et al. (1999) described two SD patients with severe loss of conceptual knowledge about objects associated with many failures to use the same items correctly.

In a follow-up study, we investigated the role of conceptual knowledge in object use with a comprehensive battery of tests devised to assess associative information, functional knowledge, and use of 20 common objects (Hodges et al., 2000). In addition to this battery of tests, the 9 SD patients were assessed on measures of general praxis and mechanical problem solving. Object use was found to be markedly impaired, and this could not be explained by problems with general praxis, since the patients performed well on copying of the meaningless gestures. Importantly, the patients' success in demonstrating the use of objects correlated strongly with their performance on naming of and semantic knowledge of the same objects. From these data, we concluded that conceptual knowledge plays a key role in object use.

The pattern of deficits seen in patients with optic aphasia is also often cited as evidence for a dissociation between impaired semantics (or in this case, impaired visual access to semantics) and preserved knowledge of object use. These patients have difficulty naming visually presented objects and pictures but can name the same items in response to tactile presentation or auditory definitions (Riddoch \& Humphreys, 1987). Most striking is the observation that patients with optic aphasia apparently can often demonstrate the appropriate use, by gesture, of objects that they fail to name upon visual confrontation. This pattern of performance, however, does not require an interpretation of preserved action semantics. Riddoch, Humphreys, Coltheart, and Funnell (1988), influenced by the work of direct perceptionists such as Marr and Gibson, suggested that these gestures were being made on the basis of nonsemantic forms of information: the perceptual attributes of the objects and/or appropriate access to a stage of processing, termed structural descriptions of objects (Humphreys \& Forde, 2000), that is intermediate between perception and semantics.

A case reported by Sirigu et al. (1991) further illustrates the influence of these nonsemantic forms of information. This associative agnosic patient (F.B.) had poor knowledge of the functional and associative attributes of objects. When asked to describe how he would use various objects and to demonstrate correct use from sight, however, his descriptions and manipulations invariably respected the mechanical affordances of the object, but not necessarily its conventional function. For instance, for the iron he said, "you hold it one hand and move it back and forth horizontally [miming the action]. Maybe you can spread glue evenly with it." Sirigu et al. argued that F.B. was able to achieve a precise analysis of the mechanical properties of the objects and that visual and or tactile inputs were able to trigger sensory motor representations, which in turn permitted appropriate action independent of 
the semantic system. It is important to emphasize, however, that even though F.B.'s manipulations invariably respected the physical affordances, they did not always lead to correct and efficient use of the objects.

In summary of this section, we conclude that there is little compelling evidence to support the hypothesis of an independent component of the semantic system representing action knowledge. There is no doubt that the ability to use objects can be disrupted when conceptual knowledge about them is preserved (Rumiati et al., 2001; Spatt et al., 2002). All such reported cases can, we think, be explained by frank nonsemantic apraxic disorders. The two possible exceptions are the patients studied by Ochipa et al. (1989) and Moreaud et al. (1998), but in these cases we question the conclusion that the patient's conceptual knowledge of objects was preserved. The other side of the putative dissociation, good object use in the face of degraded object knowledge, is a more serious issue. We have suggested above some queries regarding the evidence for this conclusion in the very few cases in which it has been suggested, but we acknowledge that it remains an unresolved issue and that the very commonly observed association (impaired object use consequent on semantic degraded conceptual knowledge) does not preclude the possibility of a genuine dissociation. Indeed, despite our preference for a theoretical position that predicts that this side of the dissociation will not be observed, our continuing research on the topic is partly motivated by this unresolved question.

\section{Affordances}

A 90-object feature database was constructed in order to identify the systematic relationships between the physical features of an object and the way it is used, to assist with a priori quantification of affordances. Affordance was determined statistically in terms of a consistent relationship across items between a structural feature (e.g., a handle of a certain type) and a specific component of use (e.g., a particular type of grip). Despite the size of this database and the number of possible correlations, there were very few that reached statistical significance. Many of the reliable correlations were either between two different structural features of an object (e.g., if the object has two handles, it is likely to have moving parts) or between a structural feature and the object's function (e.g., if the object has a sharp, serrated edge, it is likely to be used for cutting). The correlations most relevant to this study, however, were between a structural feature and the way an object is held (e.g., if the object has a handle that joins the shaft, it is likely to be held in a "standard" grasp) and between a structural feature and the way an object is moved (e.g., if the object has a wedge-shaped head, it is likely to be associated with a striking-down movement).

As a group, the patients did not achieve better performance on a subset of affordanced objects when use of these was compared with a familiarity-matched subset of objects lacking such affordances. This absence of a general group benefit applied both to overall use and to the specific component of use afforded by the object's structure. When the results were viewed as case-series data, with cases characterized by varying degrees of semantic impairment, however, it became clear that there was a reliable benefit of affordance on the specific components of use, but only for the most impaired patients. The modulation of affordance by degree of semantic impairment follows from the assumptions (1) that object use is governed principally by conceptual knowledge and (2) that affordances have a weak influence on object use. The analyses of the feature database revealed few strong correlational affordances, whose effects could be detected only for the specific component of use. It is, therefore, only when semantic memory is severely degraded that one can readily detect the influence of affordances. This proposal also explains why we found a familiarity by affordance interaction for the most impaired patients: The influence of affordances is most obvious for those objects that are relatively unfamiliar to the user.

\section{Presence of a Recipient}

It was hypothesized that having a natural recipient present might benefit the patients' object use in two ways: first, by providing a level of context and, therefore, access to further conceptual knowledge, and second, by giving clues as to the ultimate goal (i.e., the function of the object) and, therefore, encouraging trial-and-error problem-solving behavior. The patients' scores were significantly higher on the hold of the object and marginally higher on the movement when the recipient was present; however, there was no effect of recipient on orientation or overall use.

The impact of recipient, like affordance, was found to be modulated by the degree of semantic impairment. The patients with a moderate level of conceptual impairment demonstrated significantly better use with the recipient present, whereas the patients with mild and severe impairment showed no effect. Given that there was little evidence for active problem solving in any of these patients (see below) whether or not the object was presented with its recipient, it seems most likely that the recipient had its effect semantically: The combination of semantic information for the object and its recipient could boost performance, but only within a certain range of semantic deterioration. Two of the mildly impaired patients, A.N. and A.T., performed close to the normal range on assessment of single-object use, so there was little chance of measuring a positive effect when the recipient was present. Also, their conceptual knowledge was only mildly affected at this stage, so there was little room for improvement. In the moderately impaired group, the patients' semantic memory was impaired, but the combination of two mildly impoverished semantic representations (for the object and its recipient) may still be sufficient to constrain object use. In the most impaired cases, however, we suggest that conceptual representations for the object and its recipient were so impoverished as to prevent any benefit. 


\section{Familiarity}

It has been repeatedly demonstrated that familiarity is an important predictor of performance on tests involving assessment of conceptual knowledge (Bozeat et al., 2000; Funnell, 1995; Lambon Ralph \& Howard, 2000). It was not surprising, therefore, to find that familiarity also influenced performance on object use assessments. Most of the patients involved in this study had been suffering from dementia for several years, with resulting reduction in the normal variety of daily activities. This observation is confirmed by the significant difference between the ratings of familiarity obtained from the control subjects and the caregivers' ratings of how often each patient used the 36 objects selected for this study. Only personally relevant familiarity ratings predicted object use accuracy in these patients.

There are at least two possible mechanisms by which use of familiar objects is maintained. First, repeated experience with the object may boost degraded conceptual representations, which then give the patient enough information about the object to know how to use it. Alternatively, the repeated use of an object may establish a set of automatic, stereotyped responses that are triggered by that particular object and have limited reliance on semantic knowledge. These two explanations are not, in fact, mutually exclusive, and both may have a role to play.

\section{Mechanical Problem Solving}

All the patients performed within the normal range of control subjects on the Novel Tool test and the mechanical puzzles, indicating that even the patients with severe conceptual deficits had preserved mechanical-problemsolving ability. Although it is possible that the Novel Tool test (Goldenberg \& Hagmann, 1998) does not necessarily engage mechanical-problem-solving skills, relying instead on visual matching, this is not true for the mechanical puzzles (based on those designed by Ochipa et al., 1992). Despite this outcome, only 2 patients consistently used trial-and-error problem solving in the assessments of real object use, which led to improvements in movement and overall use for one patient (K.H.) and to no enhancement in the other patient (J.H.).

Why do we see such few examples of problem solving in real object use, even when the recipient is present? We suspect that the most likely explanation for this again relates to the patients' semantic impairment. Without sufficient item-specific knowledge, the patients are unable to derive the correct function for the object (as corroborated by impairments on the matching-to-function test). Knowledge of function provides the correct goal for the object, which is critical for effective problem solving to take place. It is also possible that knowledge of object properties is required for this level of object use through problem solving (Hodges et al., 2000). For example, to know that you can turn a screw by using a coin in place of the usual tool, you have to know that the metal will not bend under the twisting force required. One would certainly not try the same thing with the chocolate coins sometimes given at Christmas.
As well as enabling the delineation of the different processes involved in our everyday interaction with objects, studies of object use in SD are also relevant to debates on the streams of visual processing. From investigation of the effects of circumscribed lesions in the macaque monkey, Ungerleider and Mishkin (1982) proposed two distinct streams of visual processing: the ventral stream projecting from the primary visual cortex to the inferotemporal cortex, which enables the identification of objects, and the dorsal stream, which projects from the primary visual cortex to the posterior parietal cortex and is responsible for the localization of objects in space. Goodale and Milner (1992) reinterpreted the differences between the two streams of processing by focusing on the different requirements of the output systems that each stream serves, rather than on the different types of information handled. Furthermore, they proposed that skilled, appropriate object use is possible only through the intact functioning of both the dorsal and the ventral pathways (Milner \& Goodale, 1995). Support for the existence of these two streams of processing comes from neuropsychological dissociations between performances on tasks involving identification of objects and on those involving acting upon them. Patients with optic ataxia, who have damage to the superior portion of the posterior parietal cortex, are impaired at using visual information to reach out and grasp objects but have no difficulty recognizing or describing single objects. The patients described in this study show the opposite dissociation: They are impaired at identifying objects, because of extensive temporal lobe pathology, but can easily locate and grasp objects in space and are still able to perform mechanical-problem-solving tasks, thanks to the intact dorsal pathway. The results of this study, therefore, support the view that skilled, appropriate object use is possible only through the intact and probably interactive functioning of both the dorsal and the ventral pathways.

\section{Conclusions}

The patients with SD involved in this study were impaired both on tests of conceptual knowledge and on demonstrating the use of real objects. Furthermore, their degree of success in object use was significantly correlated with their level of semantic impairment, providing further support for the primary importance of conceptual knowledge in object use. Several other factors have also been shown to be important-namely, the affordances of objects, the presence of a recipient, and object familiarity-although in each case, this additional influence is modulated by the principal factor, the degree of semantic impairment.

\section{REFERENCES}

Bozeat, S., Lambon Ralph, M. A., Garrard, P., Patterson, K., \& Hodges J. R. (2000). Non-verbal semantic impairment in semantic dementia. Neuropsychologia, 38, 1207-1215.

Bozeat, S., Lambon Ralph, M. A., Patterson, K., \& Hodges, J. R. (2002). The influence of personal familiarity and contexts on object use in semantic dementia. Neurocase, 8, 127-134. 
Buxbaum, L. J., Schwartz, M. F., \& Carew, T. G. (1997). The role of semantic memory in object use. Cognitive Neuropsychology, 14, 219-254.

Buxbaum, L. J., Veramonti, T., \& Schwartz, M. F. (2000). Function and manipulation tool knowledge in apraxia: Knowing "what for" but not "how." Neurocase, 6, 83-97.

Folstein, M. F., Folstein, S. E., \& McHugh, P. R. (1975). "Minimental state": A practical method for grading the mental state of patients for clinicians. Journal of Psychiatric Research, 12, 189-198.

FunNell, E. (1995). From objects to properties: Evidence for spreading semantic activation in a case of semantic dementia. Memory, $\mathbf{3}$, 497-519.

FunNell, E. (2001). Evidence for scripts in semantic dementia: Implications for theories of semantic memory. Cognitive Neuropsychology, 18, 323-341.

GiBson, J. J. (1977). The theory of affordances. In R. Shaw, J. Bransford, \& N. Y. Hillsdale (Eds.), Perceiving, acting and knowing: Towards an ecological psychology (pp. 67-82). Hillsdale, NJ: Erlbaum.

GoldenberG, G. (1996). Defective imitation of gestures in patients with left and right hemisphere damage. Journal of Neurology, Neurosurgery \& Psychiatry, 61, 176-180.

Goldenberg, G., \& Hagmann, S. (1998). Tool use and mechanical problem solving in patients with apraxia. Neuropsychologia, 36, 581589.

Goodale, M. A., \& Milner, A. D. (1992). Separate visual pathways for perception and action. Trends in Neurosciences, 15, 20-25.

Graham, K. S., Lambon Ralph, M. A., \& Hodges, J. R. (1997). Determining the impact of autobiographical experience on "meaning": New insights from investigating sports related vocabulary and knowledge in two cases with semantic dementia. Cognitive Neuropsychology, 14, 801-837.

Hamanaka, T., Matsui, A., Yoshida, S., Nakanishi, M., Fujita, K., Banno, T., Murai, T., Takizawa, T., \& Hadano, K. (1996). Cerebral laterality and category-specificity in cases of semantic memory impairment with PET-findings associated with identification amnesia for familiar faces. Brain \& Cognition, 30, 368-372.

Hodges, J. R., Bozeat, S., Lambon Ralph, M. A., Patterson, K., \& SpATt, J. (2000). The role of conceptual knowledge in object use: Evidence from semantic dementia. Brain, 123, 1913-1925.

Hodges, J. R., Graham, N., \& Patterson, K. (1995). Charting the progression in semantic dementia: Implications for the organisation of semantic memory. Memory, 3, 463-495.

Hodges, J. R., Patterson, K., Oxbury, S., \& Funnell, E. (1992). Semantic dementia: Progressive fluent aphasia with temporal lobe atrophy. Brain, 115, 1783-1806.

Hodges, J. R, Spatt, J., \& Patterson, K. (1999). What and how: Evidence for the dissociation of object knowledge and mechanical problem solving skills in the human brain. Proceedings of the National Academy of Sciences, 96, 775-784.

Howard, D., \& Patterson, K. (1992). Pyramids and palm trees: A test of semantic access from pictures and words. Bury St. Edmunds, U.K.: Thames Valley Test Company.

Humphreys, G. W., \& Forde, E. M. E. (2000). Hierarchies, similarity and interactivity in object recognition: "Category-specific" neuropsychological deficits. Behavioural \& Brain Sciences, 24, 453-476.

KofFKA, K. (1935). Principles of Gestalt psychology. New York: Harcourt, Brace \& World.

Lambon Ralph, M. A., Graham, K. S., Ellis, A., \& Hodges, J. R. (1998). Naming in semantic dementia: What matters? Neuropsychologia, 36, 775-784.

Lambon Ralph, M. A., \& Howard, D. (2000). Gogi aphasia or semantic dementia? Simulating and assessing poor verbal comprehension in a case of progressive fluent aphasia. Cognitive Neuropsychology, 17, 437-465.

Lauro-Grotto, R., Piccini, C., \& Shallice, T. (1997). Modalityspecific operations in semantic dementia. Cortex, 33, 593-622.
Milner, A. D., \& Goodale, M. A. (1995). The visual brain in action. Oxford: Oxford University Press.

Moreaud, O., Charnallet, A., \& Pellat, J. (1998). Identification without manipulation: A study of the relations between object use and semantic memory. Neuropsychologia, 36, 1295-1301.

Mummery, C. J., Patterson, K., Price, C. J., Ashburner, J., Frackowick, R. S., \& Hodges, J. R. (2000). A voxel based morphometry study of semantic dementia: The relation of temporal lobe atrophy to cognitive deficit. Annals of Neurology, 47, 36-45.

Mummery, C. J., Patterson, K., Wise, R. J. S., Price, C. J., \& Hodges, J. R. (1999). Disrupted temporal lobe connections in semantic dementia. Brain, 122, 61-73.

NeISSER, U. (1994). Multiple systems: A new approach to cognitive theory. European Journal of Cognitive Psychology, 6, 225-241.

Ochipa, C., Rothi, L. J. G., \& Heilman, K. M. (1989). Ideational apraxia: A deficit in tool selection and use. Annals of Neurology, 25, 190-193.

Ochipa, C., Rothi, L. J. G., \& Heilman, K. M. (1992). Conceptual apraxia in Alzheimer's disease. Brain, 115, 1061-1071.

RAVEN, J. C. (1962). Coloured progressive matrices Sets A, AB, B. London: Lewis.

RAVEN, J. C. (1965). Advanced progressive matrices Sets I and II. London: Lewis.

REY, A. (1941). L'examen psychologiquedans les cas d'encephalopathie traumatique. Archives de Psychologie, 28, 286-340.

Riddoch, M. J., \& Humphreys, G. W. (1987). A case of integrative visual agnosia. Brain, 110, 1431-1462.

Riddoch, M. J., Humphreys, G. W., Coltheart, M., \& Funnell, E. (1988). Semantic systems or system? Neuropsychological evidence reexamined. Cognitive Neuropsychology, 5, 3-25.

Rumiati, R. I., Zanini, S., Vorano, L., \& Shallice, T. (2001). A form of ideational apraxia as a selective deficit of contention scheduling. Cognitive Neuropsychology, 18, 617-642.

Sirigu, A, Duhamel, J., \& Poncet, M. (1991). The role of sensorimotor experience in object recognition: A case of multimodal agnosia. Brain, 114, 2555-2573.

Snowden, J. S., Goulding, P. J., \& Neary, D. (1989). Semantic dementia: A form of circumscribed cerebral atrophy. Behavioural Neurology, 2, 167-182.

Snowden, J. S., Griffiths, H., \& Neary, D. (1994). Semantic dementia: Autobiographical contribution to preservation of meaning. Cognitive Neuropsychology, 11, 265-288.

SNOWDEN, J. S., NEARY, D., \& MANn, D. M. A. (1996). Fronto-temporal lobar degeneration: Fronto-temporal dementia, progressive aphasia, semantic dementia. New York: Churchill Livingstone.

Spatt, J., Bak, T., Bozeat, S., Patterson, K., \& Hodges, J. R. (2002). Apraxia, mechanical problem solving and semantic knowledge: Contributions to object usage in corticobasal degeneration. Journal of Neurology, 249, 601-608.

UNGERLEIDER,L. G., \& MishKIN, M. (1982). Two cortical visual systems. In D. J. Ingle, M. A. Goodale, \& R. J. W. Mansfield (Eds.), Analysis of visual behavior (pp. 549-586). Cambridge, MA: MIT Press.

WARRINGTON, E. K. (1975). Selective impairment of semantic memory. Quarterly Journal of Experimental Psychology, 27, 635-657.

WARrington, E. K., \& JAMES, M. (1986). Visual object recognition in patients with right hemisphere lesions: Axes or features. Perception, 15, 355-366.

WeCHSLER, D. A. (1981). Wechsler Adult Intelligence Scale-Revised: Test manual. New York: Psychological Corporation.

Zangwill, O. L. (1960). L'apraxie idéatorie. Nerve Neurology, 106, 595-603.

(Manuscript received October 15, 2001; revision accepted for publication April 12, 2002.) 